\title{
Metal-cavity surface-emitting microlaser at room temperature
}

Cite as: Appl. Phys. Lett. 96, 251101 (2010); https://doi.org/10.1063/1.3455316

Submitted: 14 April 2010 • Accepted: 24 May 2010 • Published Online: 21 June 2010

Chien-Yao Lu, Shu-Wei Chang, Shun Lien Chuang, et al.

\section{ARTICLES YOU MAY BE INTERESTED IN}

An electrical injection metallic cavity nanolaser with azimuthal polarization Applied Physics Letters 102, 041110 (2013); https://doi.org/10.1063/1.4775803

Whispering-gallery mode microdisk lasers

Applied Physics Letters 60, 289 (1992); https://doi.org/10.1063/1.106688

Hybrid gap plasmon GaAs nanolasers

Applied Physics Letters 111, 261107 (2017); https://doi.org/10.1063/1.5008320

四 QBLOX

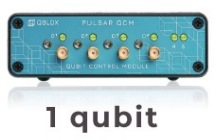

Shorten Setup Time Auto-Calibration More Qubits

Fully-integrated Quantum Control Stacks Ultrastable DC to $18.5 \mathrm{GHz}$ Synchronized $<<1 \mathrm{~ns}$ ultralow noise

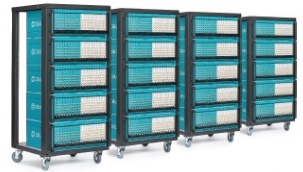

100s qubits

visit our website > 


\title{
Metal-cavity surface-emitting microlaser at room temperature
}

\author{
Chien-Yao Lu, ${ }^{1}$ Shu-Wei Chang, ${ }^{1}$ Shun Lien Chuang, ${ }^{1, a)}$ Tim D. Germann, ${ }^{2}$ and \\ Dieter Bimberg ${ }^{2}$ \\ ${ }^{1}$ Department of Electrical and Computer Engineering, University of Illinois at Urbana-Champaign, \\ 1406 West Green Street, Urbana, Illinois 61801, USA \\ ${ }^{2}$ Institut für Festkörperphysik, Technische Universität Berlin, Sekretariat EW 5-2, Hardenbergstrase 36, \\ 10623 Berlin, Germany
}

(Received 14 April 2010; accepted 24 May 2010; published online 21 June 2010)

\begin{abstract}
We propose and realize a substrate-free metal-cavity surface-emitting microlaser with both top and sidewall metal and a bottom distributed Bragg reflector as the cavity structure. The transfer-matrix method is used to design the laser structure based on the round-trip resonance condition inside the cavity. The laser is $2.0 \mu \mathrm{m}$ in diameter and $2.5 \mu \mathrm{m}$ in height, and operates at room temperature with continuous-wave mode. Flip-bonding the device to a silicon substrate with a conductive metal provides efficient heat removal. A high characteristic temperature about $425 \mathrm{~K}$ is observed from 10 to $27{ }^{\circ} \mathrm{C}$. (C) 2010 American Institute of Physics. [doi:10.1063/1.3455316]
\end{abstract}

Metals, with a negative permittivity at optical frequency, can be engineered to support a surface-plasmon wave and confine the optical field within a skinny region near the metal/dielectric interface. ${ }^{1,2}$ With possible energy localization near the metal/dielectric interface, metal cavities become a promising resonance structure in the subwavelength regime. $^{3}$ Despite its large ohmic loss at optical frequencies, lasing in metal cavities is realizable even for a moderate quality factor. ${ }^{4,5}$ Although these quality factors are not higher than those of conventional dielectric cavities, the photon life time becomes shorter, and therefore, a higher modulation speed is expected. Moreover, an enhanced bandwidth with the shrinkage of the aperture in oxide-confined vertical-cavity surface emitting lasers (VCSELs) due to the smaller mode volume has been reported. ${ }^{6}$ The mode volume of the metal-coated devices can be even smaller and potentially leads to a higher modulation bandwidth. Another superior property originates from the high thermal conductivity of the metal, which removes the heat that would degrade the material and device performance, especially for ultrasmall devices under high-density current injection, like VCSELs.

Room-temperature operation of a metal-coated laser with bottom emission has recently been demonstrated at pulsed current injection. ${ }^{5}$ Several interesting effects have been discovered, including surface-plasmon-mode lasing and high group indices due to large metal dispersions. However, when the device size is comparable or even smaller than the wavelength, several issues such as the small-aperture effect and resonance shift due to the change in the effective index should be taken into consideration. ${ }^{7}$

In this letter, we propose the design, show our fabrication, and demonstrate a metal-cavity surface-emitting laser with metal and distributed Bragg reflector (DBR) as the feedback structures, surrounded by a metal cavity as the sidewall. The emission from the top of devices can be more easily coupled to fibers or lens. Moreover, the metal serves as a broadband high-reflectivity mirror, as well as the substrate and heat sink for these devices. From an application viewpoint, this configuration eases the device integration to sili-

\footnotetext{
a) Author to whom correspondence should be addressed. Electronic mail: s-chuang@illinois.edu.
}

con or other substrates that could potentially be used as the platform for optical interconnects in the terabit range. In terms of fabrication, using top metal as the feedback mirror prevents the deep etching, which is usually challenging for submicron or nanodevices. Also, by coating the sidewall with metal, the diffraction loss from the small aperture and sidewall roughness can be reduced.

The epistructure is composed of a p-type $\operatorname{GaAs}(20 \mathrm{~nm}) / \mathrm{Al}_{0.2} \mathrm{Ga}_{0.8} \mathrm{As}(96 \mathrm{~nm})$ cladding and an n-type 17.5-pair alternating $\mathrm{Al}_{0.9} \mathrm{Ga}_{0.1} \mathrm{As} / \mathrm{Al}_{0.15} \mathrm{Ga}_{0.85} \mathrm{As}$ quarterwavelength DBR. The active medium consists of a GaAs $/ \mathrm{Al}_{0.2} \mathrm{Ga}_{0.8} \mathrm{As}$ multiple quantum-well (MQW) structure (14 wells and 15 barriers). An InGaP etch-stop layer is inserted between DBR and GaAs substrate for substrate removal. The thickness of each layer was determined based on the transfer-matrix method. ${ }^{8}$ To include the metal effect and modal confinement properties, the modal effective index of the fundamental $\mathrm{HE}_{11}$ mode is used instead of bulk semiconductor refractive index. Due to the plasma damping in metals at optical frequencies, the complex permittivity is introduced in the layer design. ${ }^{8,9}$ The thickness of the insulator layer between metal and sidewall was also optimized ${ }^{10}$ and taken into account in the modal effective index. The total roundtrip phase shift is ${ }^{8}$

$$
\begin{aligned}
\Phi= & 2 k_{0} n_{\mathrm{GaAs}} d_{\mathrm{GaAs}}+2 k_{0} n_{\mathrm{Al}_{0.2} \mathrm{Ga}_{0.8} \mathrm{As}} d_{\mathrm{Al}_{0.2} \mathrm{Ga}_{0.8} \mathrm{As}} \\
& +2 k_{0} n_{\mathrm{QW}} d_{\mathrm{QW}}+\phi_{\text {metal }}+\phi_{\mathrm{DBR}},
\end{aligned}
$$

where $n_{\mathrm{GaAs}}$ and $n_{\mathrm{Al}_{0.2} \mathrm{Ga}_{0.8} \mathrm{As}}$ are the effective indices of the $\mathrm{HE}_{11}$ mode in these two materials; $d_{\mathrm{GaAs}}$ and $d_{\mathrm{Al}_{0.2} \mathrm{Ga}_{0.8} \mathrm{As}}$ are thicknesses of top p-type GaAs and $\mathrm{Al}_{0.2} \mathrm{Ga}_{0.8}$ As layers; $n_{\mathrm{QW}}$ is the effective index of the $\mathrm{HE}_{11}$ mode calculated for the metal-cavity waveguide using an average refractive index of the MQW region; $d_{\mathrm{QW}}$ is the total thickness of the active region; $k_{0}$ is the free space propagation constant; and $\phi_{\text {metal }}$ and $\phi_{\mathrm{DBR}}$ are the phase shifts in the complex reflection coefficients of the top metal mirror and bottom DBR, respectively. The thickness of active region was tuned to match the round-trip resonance condition $(\phi=2 m \pi, m=0,1,2, \ldots)$ of the bottom DBR and top metal for the formation of standing wave inside the cavity. The phase shift from top metal $\phi_{\text {metal }}$ was estimated by Fresnel formula with complex wavelength- 

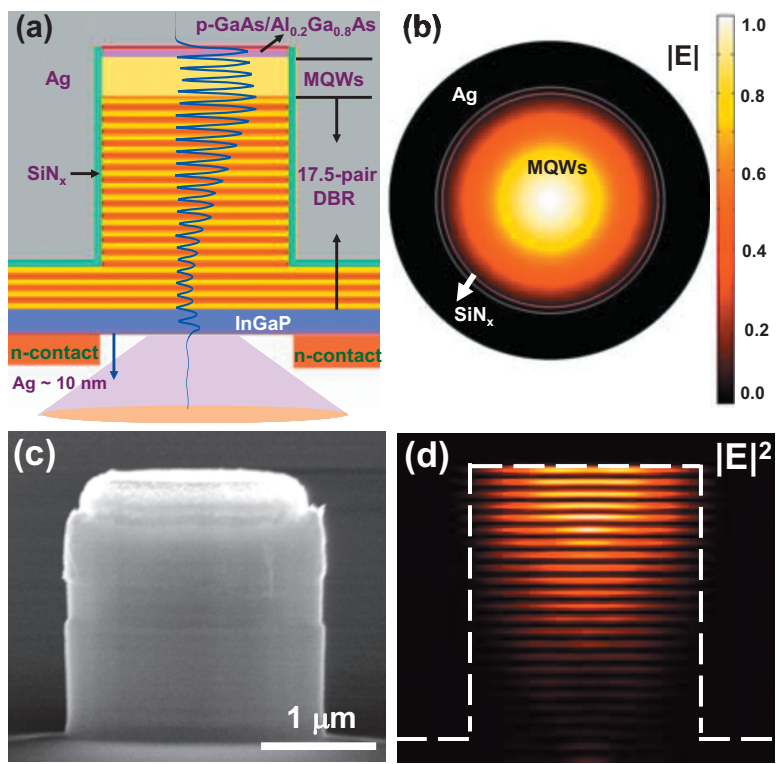

FIG. 1. (Color) (a) Schematics of the microlaser. The blue line represents the magnitude of the optical field $|\mathbf{E}|$. The laser cavity is formed with both top and sidewall metal and a bottom DBR. The laser is $2.0 \mu \mathrm{m}$ in diameter and $2.5 \mu \mathrm{m}$ in height with the substrate removed. (b) The corresponding $\mathrm{HE}_{11}$ mode pattern. (c) A scanning electron micrograph of the fabricated cavity with the $\mathrm{SiN}_{\mathrm{x}}$ surrounding before metal coating. (d) The field pattern $|\mathbf{E}|^{2}$ of the device simulated by FDTD.

dependent permittivities of metal and semiconductor. ${ }^{9,11}$ Also, with the additional phase shift $\phi_{\mathrm{DBR}}$ from the bottom $\mathrm{DBR}$, the round-trip resonance condition can be fulfilled inside the cavity. In our design, the active region thickness was chosen to be $426.0 \mathrm{~nm}$, corresponding to a standing-wave pattern of 3.5 antinodes in the active region. Compared to designs of conventional VCSELs, ${ }^{12}$ which consider only the material dielectric constants without the modal properties, our design, due to the small device size and high permittivity contrast between metals and dielectrics, brings about more flexibility for the resonance tuning by adjusting cavity dimensions. Figure 1(a) shows the device structure and standing wave pattern (magnitude of the electric field $|\mathbf{E}|$ ) inside the cavity. The $\mathrm{HE}_{11}$ mode has the highest $Q$ factor and closest resonance wavelength to the experimental data. The corresponding modal profile at the anitnode is shown in Fig. 1(b). The designed resonance is found to be at $869.2 \mathrm{~nm}$ with a quality factor of 505. The threshold material gain $g_{t h}$ can be estimated by the following formula: ${ }^{13}$

$$
g_{t h}=\frac{\omega}{\Gamma_{E} v_{g} Q},
$$

where $\Gamma_{E}$ is the energy confinement factor, $Q$ is the cavity quality factor, $\omega$ is the optical angular frequency, and $v_{g}$ is the material group velocity which can be estimated by the speed of light divided by the group index of GaAs. The estimated value of the threshold gain is around $8400 \mathrm{~cm}^{-1}$.

The epistructure was grown by industry standard metalorganic vapor-phase epitaxy using a commercial AIXTRON 200/4 reactor and partly alternative precursors. ${ }^{14}$ A silicon nitride $\left(\mathrm{SiN}_{\mathrm{x}}\right)$ layer was deposited and patterned as the mask for the following dry etching. Reactive ion etching on the epiwafer was performed with chlorine-based reaction gas. A thin $\mathrm{SiN}_{\mathrm{x}}$ layer was conformally deposited after the removal of the top silicon-nitride mask. Planarization was used to
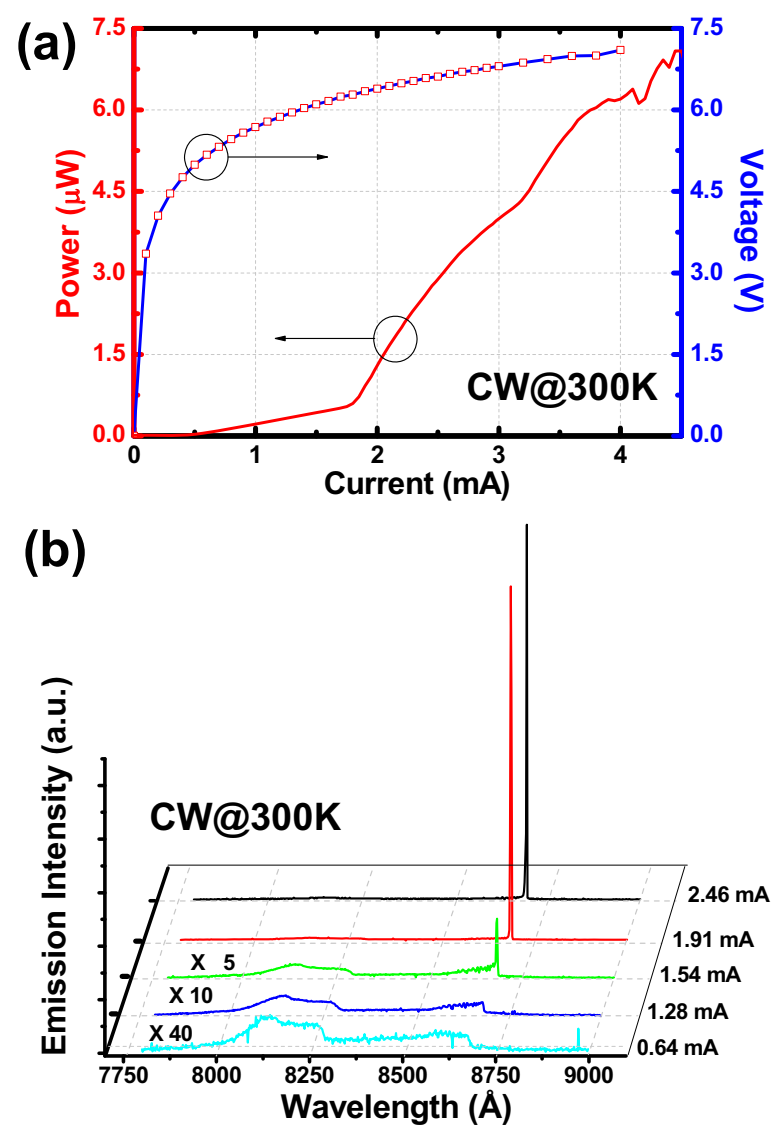

FIG. 2. (Color online) (a) The I-V and L-I curves measured at room temperature $(300 \mathrm{~K})$ under dc current injection. The L-I curve shows a threshold of $1.66 \mathrm{~mA}$. (b) The corresponding current-dependent spectra. Below threshold, a broad spontaneous emission spectrum was observed. The lasing peak around $868 \mathrm{~nm}$ appeared when the injection current exceeded the threshold value.

expose the top of the post covered by silicon nitride, which was then removed to facilitate the following contact formation. E-beam evaporation was used to uniformly coat the device with $200 \mathrm{~nm}$ silver and $50 \mathrm{~nm}$ gold layers for oxidation protection. The sample was then flip-bonded to a goldcoated silicon receptor wafer. The GaAs substrate was completely removed by a mixture of citric acid and hydrogen peroxide. N-type contacts $(\mathrm{AuGe} / \mathrm{Ni} / \mathrm{Au})$ were formed and defined on the InGaP etching-stop layer. Figure 1(c) shows the fabricated $2.0-\mu \mathrm{m}$-diameter cavity before silver coating. A full-structure simulation was carried out by finite difference time domain (FDTD) method with the real device size and geometry. According to the field pattern in Fig. 1(d), most of the energy is stored in the active region with an exponentially decaying tail into the DBR as a consequence of the successive reflections from DBR periods. The mode pattern shows that the mode is indeed bouncing back and forth between top metal and bottom DBRs and confined by the metal sidewall.

The measurements were performed at $300 \mathrm{~K}$ with a thermoelectrically-cooled heat sink on which the devices were mounted. The emission was collected by an objective lens, dispersed by a $1.25 \mathrm{~m}$ monocromator, and detected by a liquid-nitrogen-cooled germanium detector. The current versus voltage (I-V) and light output power versus current (L-I) curves under $\mathrm{CW}$ operation shown in Fig. 2(a) indicate a turn-on threshold at around $1.66 \mathrm{~mA}$, beyond which a lasing peak emerges at $868 \mathrm{~nm}$ with a gradual redshift as the driv- 

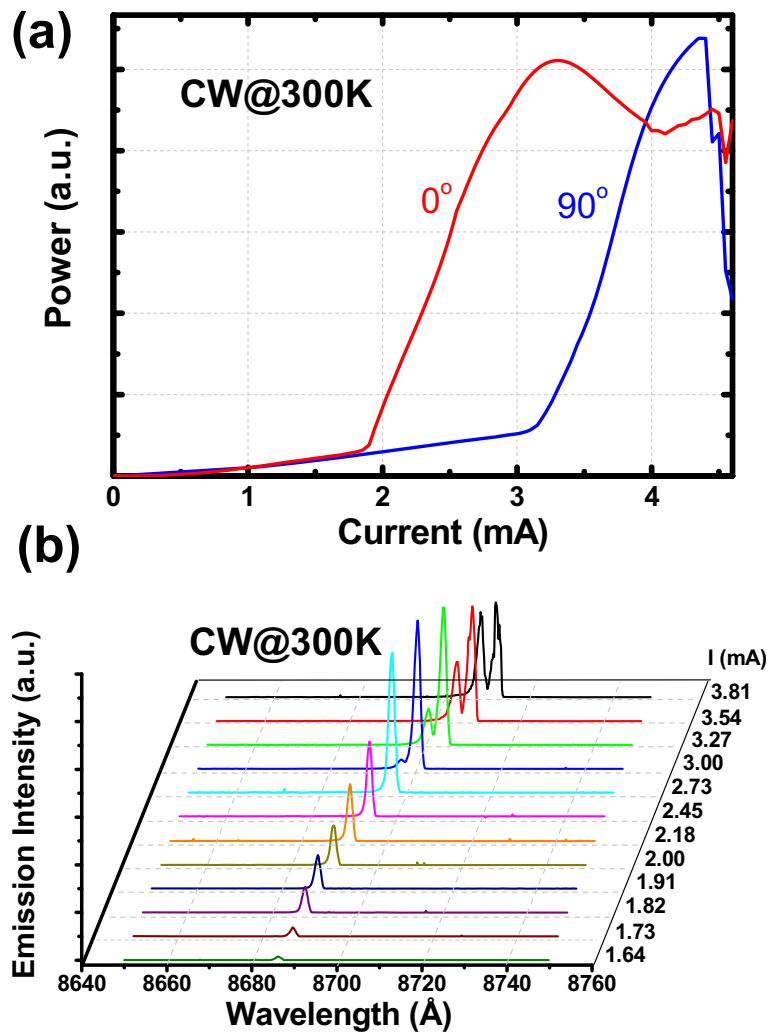

FIG. 3. (Color online) (a) The polarization-resolved L-I curves indicate two modes due to the splitting of two degenerate $\mathrm{HE}_{11}$ modes. A clear onset of the second mode around $3.30 \mathrm{~mA}$ was observed. (b) The spectral evolution of the two lasing peaks with increasing current. The second peak emerged above its threshold. A clear redshift due to heat was observed when the current increased. All data were taken with $\mathrm{CW}$ operation at $300 \mathrm{~K}$.

ing current increases [Fig. 2(b)]. The output power increases from $0.5 \mu \mathrm{W}$ near threshold to over $7 \mu \mathrm{W}$ at $4.5 \mathrm{~mA}$. We also observed a second mode emerging at a wavelength 0.4 $\mathrm{nm}$ shorter than the first lasing peak. This additional mode comes from the splitting of $\mathrm{HE}_{11}$ mode into two orthogonal and quasilinearly polarized modes, most likely due to a small anisotropy of the waveguide structure. ${ }^{15}$ As shown in Fig. 2(a), the kink in the L-I curve around $3.2 \mathrm{~mA}$ corresponds to the onset of the second peak. To identify the origin of the second peak, we show the polarization-resolved L-I curves of individual modes in Fig. 3(a). From Fig. 3(b), the onset of the second lasing peak reflects the kink in the L-I curve shown in Fig. 2(a). Near threshold, the linewidth of the lasing peak is estimated to be around $1.5 \mathrm{~nm}$ (@ $1.45 \mathrm{~mA}$ ), which gives a $Q$ factor of 580 and agrees quantitatively with that of our transfer-matrix estimation for a cold cavity. The narrowest setup-resolvable linewidth is $67 \mathrm{pm}$ at $2.73 \mathrm{~mA}$, obtained just below the threshold of the second peak. The threshold current density was estimated to be $52.8 \mathrm{kA} / \mathrm{cm}^{2}$. With the lossy metal coating and high radiation loss from the moderate reflectivity (about 0.93) of the silver mirror, the threshold current density is high. The detuning of the cavity resonance wavelength from the band-edge wavelength $(845$ $\mathrm{nm}$ ) of the MQW active region and a nonuniform distribution of carriers in the 14 quantum wells may be additional reasons for the high threshold. Further improvements, such as reducing threshold by tuning the resonance toward the peak gain wavelength and size reduction to submicron diameter, are in progress.
From the measured L-I curves and the threshold currents for temperature from 10 to $27{ }^{\circ} \mathrm{C}$, we obtained a characteristic temperature $T_{0}$ of $425 \mathrm{~K}$. This characteristic temperature is considerably higher than the reported values ${ }^{16}$ for semiconductor quantum-well lasers with bulk substrates. In contrast to conventional top-and-bottom-DBR lasers in which the resonance shifts due to the effect of temperature on DBR phase and reflectivity, ${ }^{17}$ the stability of our laser may be due to the broadband high-reflectivity window provided by top metallic mirror, which makes the resonance less sensitive to temperature. Also, we believe that the surrounding metal and flip bonding remove heat more efficiently and keep the operation temperature more stable.

In summary, we have demonstrated the design and implementation of room-temperature $\mathrm{CW}$ substrate-free metal-cavity surface-emitting microlasers with a top and sidewall metal and a bottom DBR as feedback structures. The modal properties of the guided modes have been incorporated into the design. The devices showed good thermal stability due to metal coating and flip bonding to silicon. These metal-cavity microlasers provide a great potential for next generation massively parallel optical interconnects without crosstalk among laser elements.

This work at UIUC was supported partially by DARPA W911NF-07-1-0314. S.L.C. also thanks the support by the Humboldt Research Award. The work at TUB was supported by Deutsche Forschungsgemeinschaft in the frame of Grant No. SFB 787.

${ }^{1}$ W. L. Barnes, A. Dereux, and T. W. Ebbesen, Nature (London) 424, 824 (2003)

${ }^{2}$ H. T. Miyazaki and Y. Kurokawa, Phys. Rev. Lett. 96, 097401 (2006).

${ }^{3}$ R. Zia, M. D. Selker, P. B. Catrysse, and M. L. Brongersma, J. Opt. Soc. Am. A 21, 2442 (2004).

${ }^{4}$ M. T. Hill, Y. S. Oei, B. Smalbrugge, Y. Zhu, T. de Vries, P. J. van Veldhoven, F. W. M. van Otten, T. J. Eijkemans, J. P. Turkiewicz, H. de Waardt, E. J. Geluk, S. H. Kwon, Y. H. Lee, R. Nötzel, and M. K. Smit, Nat. Photonics 1, 589 (2007)

${ }^{5}$ M. T. Hill, M. Marell, E. S. P. Leong, B. Smalbrugge, Y. Zhu, M. Sun, P. J. van Veldhoven, E. J. Geluk, F. Karouta, Y. Oei, R. Nötzel, C. Z. Ning, and M. K. Smit, Opt. Express 17, 11107 (2009).

${ }^{6}$ A. Mutig, S. A. Blokhin, A. M. Nadtochiy, G. Fiol, J. A. Lott, V. A. Shchukin, N. N. Ledentsov, and D. Bimberg, Appl. Phys. Lett. 95, 131101 (2009).

${ }^{7}$ A. Yariv and P. Yeh, Potonics: Optical Electronics in Modern Communications, 6th ed. (Oxford University Press, New York, 2007).

${ }^{8}$ S. L. Chuang, Physics of Photonic Devices, 2nd ed. (Wiley, New York, 2009).

${ }^{9}$ Handbook of Optical Constants of Solids, edited by E. D. Palik (Adademic, New York, 1985).

${ }^{10}$ A. Mizrahi, V. Lomakin, B. A. Slutsky, M. P. Nezhad, L. Feng, and Y. Fainman, Opt. Lett. 33, 1261 (2008).

${ }^{11}$ S. Adachi, J. Appl. Phys. 58, R1 (1985).

${ }^{12}$ L. W. Tu, E. F. Shubert, R. F. Kopf, G. J. Zydzik, M. Hong, S. N. G. Chu, and J. P. Mannaerts, Appl. Phys. Lett. 57, 2045 (1990).

${ }^{13}$ S. W. Chang and S. L. Chuang, IEEE J. Quantum Electron. 45, 1014 (2009).

${ }^{14}$ T. D. Germann, A. Strittmatter, U. W. Pohl, D. Bimberg, J. Rautiainen, M. Guina, and O. G. Okhotnikov, J. Cryst. Growth 310, 5182 (2008).

${ }^{15}$ K. D. Choquette, R. P. Schneider, Jr., K. L. Lear, and R. E. Leibenguth, IEEE J. Sel. Top. Quantum Electron. 1, 661 (1995).

${ }^{16}$ WDM Technology: Active Optical Components, edited by A. K. Dutta, N. K. Dutta, and M. Fujiwara (Academic, New York, 2002), Chap. 5.

${ }^{17}$ Vertical Cavity Surface Emitting Lasers, edited by C. W. Wilmsen, H. Temkin, and L. A. Coldren (Cambridge University Press, Cambridge, 2001), Chap. 5. 